\title{
Improvements of Beam Structural Modelling in Hydroelasticity of Ultra Large Container Ships
}

Article · January 2011

DOI: 10.1115/OMAE2011-49337

CITATIONS

3

3 authors, including:

\section{Nikola Vladimir}

University of Zagreb

112 PUBLICATIONS 210 CITATIONS

SEE PROFILE
READS

34

Marko Tomic

University of Zagreb

41 PUBLICATIONS 123 CITATIONS

SEE PROFILE 


\section{OMAE2011-49ロपा}

\section{IMPROVEMENTS OF BEAM STRUCTURAL MODELLING IN HYDROELASTICITY OF ULTRA LARGE CONTAINER SHIPS}

\author{
Ivo Senjanović \\ University of Zagreb, Faculty of Mechanical \\ Engineering and Naval Architecture \\ I. Lucica 5, 10000 Zagreb, Croatia \\ Šime Malenica \\ Bureau Veritas \\ 67/71 Boulevard du Châteu \\ 92200 Neuilly-sur-Seine, France
}

\author{
Nikola Vladimir \\ University of Zagreb, Faculty of Mechanical \\ Engineering and Naval Architecture \\ I. Lucica 5, 10000 Zagreb, Croatia \\ Marko Tomić \\ University of Zagreb, Faculty of Mechanical \\ Engineering and Naval Architecture \\ I. Lucica 5, 10000 Zagreb, Croatia
}

\begin{abstract}
Increase in global ship transport induces building of Ultra Large Container Ships (ULCS), which have a capacity up to 14000 TEU with length up to $400 \mathrm{~m}$, without changes of the operational requirements (speed around 27 knots). Natural frequencies of such ships can fall into the range of encounter frequencies in an ordinary sea spectrum. Present Classification Rules for ship design and construction don't cover such conditions completely and hydroelastic analysis of ULCS seems to be the appropriate solution for analysis of their response in waves. This paper deals with numerical procedure for ship hydroelastic analysis with particular emphasis on improvements of the present beam structural model. The structural model represents a constitutive part of hydroelastic mathematical model and generally it can be formulated either as 1D FEM or 3D FEM model. For the preliminary design stage hydroelastic model derived by coupling $1 \mathrm{D}$ FEM structural model and 3D BEM hydrodynamic one seems to be an appropriate choice. Within the paper the importance of hydroelastic approach and methodology of hydroelastic analysis are elaborated. Further on, structural model based on advanced beam theory is described in details. The improvements include taking into account shear influence on torsion, contribution of bulkheads to hull stiffness as well as determination of effective stiffness of engine room structure. Along with that, hydrodynamic and hydrostatic models are presented in a condensed form. Numerical example, which includes complete hydroelastic analysis of a large container ship, is also added. In this case, validation of 1D FEM model is checked by correlation analysis with the vibration response of the fine 3D FEM model. The procedure related to determination of engine room effective stiffness is checked by 3D FEM analysis of ship-like pontoon which has been made according to the considered ship characteristics.
\end{abstract}

\section{INTRODUCTION}

Modern sea transport requires design and building of Ultra Large Container Ships (ULCS), which are relatively flexible and fast vessels. The classical theories for determination of ship motions and wave loads, as for example [1], are based on the assumption that the ship hull is a rigid body. Usually, the wave load obtained according to these theories is imposed to the elastic 3D FEM model of ship structure in order to analyze global strength, as well as local strength with stress concentrations related to fatigue. Although the above approach is good enough for ships with closed cross-section and ordinary hatch openings such as tankers, bulk carriers or general cargo ships, it is not reliable as it should be for ultra large container ships due to mutual influence of the wave load and structure response [2, 3]. Therefore, a more reliable solution requires analysis of wave load and ship vibration as a coupled hydroelastic problem [4]. This is very important in case of impulsive loads such as ship slamming and induced whipping.

Numerical procedure for ship hydroelastic analysis requires definition of structural model, ship and cargo mass distributions, and geometrical model of ship wetted surface $[3, \underline{5,} \underline{6,7]}$. Firstly, dry natural vibrations have to be calculated, and after that modal hydrostatic stiffness, modal added mass, damping and modal wave load are determined. Finally, wet natural vibrations as well as the transfer functions (RAO) for determining ship structural response to wave excitation are obtained [6, 7].

This paper on the first place summarizes the improvements of the structural model based on the beam and thin-walled girder theories for calculation of dry natural vibrations of container ships $[8,9]$. First improvement is related to taking into account shear influence on torsion as an analogy with shear influence on bending [10]. The second one includes contribution of transverse bulkheads to hull stiffness [11]. Third improvement is related to influence of engine 
room structure on hull stiffness of ULCS [12], and it is considered in details in separate chapter. Also, short description of hydrostatic and hydrodynamic submodels, and hydroelastic model is given. Finally, the results of hydroelastic analysis of 7800 TEU container ship are shown. It should be mentioned that the applied numerical procedure is verified earlier, by correlation analysis with experimental results for a flexible segmented barge, for which test results are available [13, 14, 15]. Details of the verification are presented in the literature $[2,3,9]$.

\section{BEAM STRUCTURAL MODEL}

\subsection{Review of an advanced beam theory}

Referring to the Timoshenko's flexural beam theory, the total beam deflection, $w$, consists of the bending deflection, $w_{b}$, and the shear deflection, $w_{s}$, i.e., [16], Figure 1

$w=w_{b}+w_{s}, \quad w_{s}=-\frac{E I_{b}}{G A_{s}} \frac{\partial^{2} w_{b}}{\partial x^{2}}$,

where $E$ and $G$ are the Young's and shear modulus, respectively, while $I_{b}$, and $A_{s}$ are the moment of inertia of cross-section and shear area, respectively. The angle of cross-section rotation is caused by the bending deflection

$\varphi=\frac{\partial w_{b}}{\partial x}$.

The cross-sectional forces are the bending moment and the shear force

$M=-E I_{b} \frac{\partial^{2} w_{b}}{\partial x^{2}}, \quad Q=G A_{s} \frac{\partial w_{s}}{\partial x}=-E I_{b} \frac{\partial^{3} w_{b}}{\partial x^{3}}$.

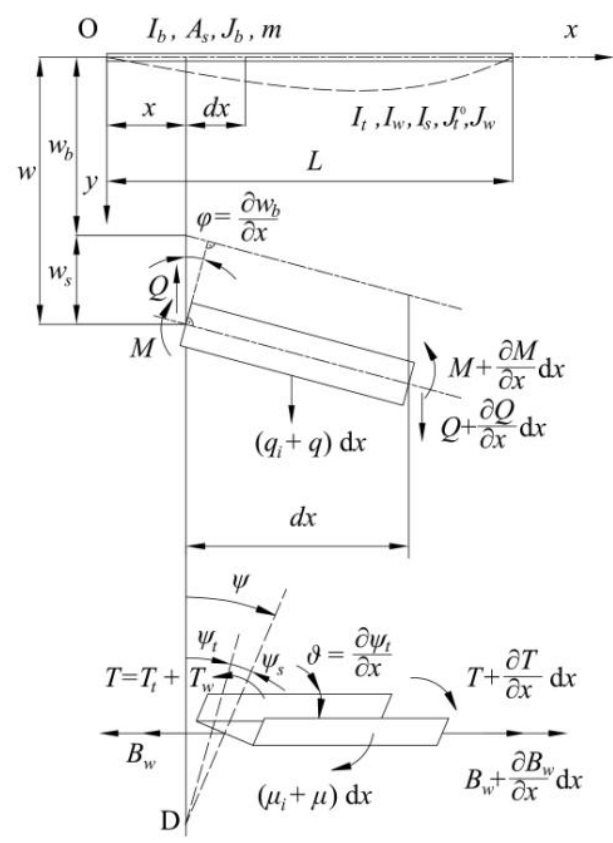

Figure 1. Beam bending and torsion

Concerning torsion, the total twist angle, $\psi$, consists of the pure twist angle, $\psi_{t}$, and the shear contribution, $\psi_{s}$, i.e., Figure 1 , and referring to the analogy of torsion and bending [10], the shear angle depends on the twist angle, similarly to Eq. (1)

$\psi=\psi_{t}+\psi_{s}, \quad \psi_{s}=-\frac{E I_{w}}{G I_{s}} \frac{\partial^{2} \psi_{t}}{\partial x^{2}}$,

where $I_{w}$ is the warping modulus and $I_{s}$ is the shear inertia modulus. The second beam displacement, which causes warping of cross-section (similarly to the cross-section rotation due to bending) is a variation of the pure twist angle

$\vartheta=\frac{\partial \psi_{t}}{\partial x}$

The sectional forces include the total torque, $T$, which consists of pure torsional torque, $T_{t}$, and the warping torque $T_{w}$ i.e.

$T=T_{t}+T_{w}$,

where

$T_{t}=G I_{t} \frac{\partial \psi_{t}}{\partial x}, \quad T_{w}=G I_{s} \frac{\partial \psi_{s}}{\partial x}=-E I_{w} \frac{\partial^{3} \psi_{t}}{\partial x^{3}}$,

and the bimoment given by

$B_{w}=E I_{w} \frac{\partial^{2} \psi_{t}}{\partial x^{2}}$

\subsection{Contribution of transverse bulkheads}

This problem for container ships is extensively analyzed in [11], where torsional modulus of ship cross-section is increased proportionally to the ratio of bulkhead strain energy and strain energy of corresponding hull portion. The bulkhead is considered as an orthotropic plate with very strong stool [17]. Bulkhead strain energy is determined for the given warping of cross-section as a boundary condition. The warping causes bulkhead screwing and bending. Here, only the review of the final results is presented. Bulkhead deflection (axial displacement) is given by the following formula, Figure 2 :

$u(y, z)=-y\left\{(z-d)+\left[1-\left(\frac{y}{b}\right)^{2}\right] \frac{z^{2}}{H}\left(2-\frac{z}{H}\right)\right\} \psi^{\prime}$,

where $H$ is the ship height, $b$ is one half of bulkhead breadth, $d$ is the distance of warping centre from double bottom neutral line, $y$ and $z$ are transverse and vertical coordinates, respectively, and $\psi^{\prime}$ is the variation of twist angle.

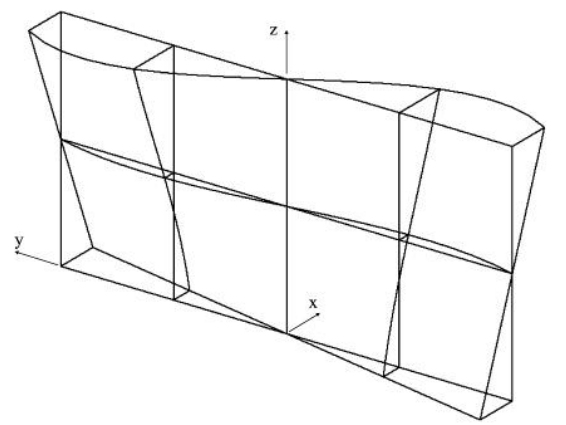

Figure 2. Shape of bulkhead deformation 
The bulkhead grillage strain energy includes vertical and horizontal bending with contraction and torsion [11].

$$
\begin{aligned}
& U_{g}=\frac{1}{1-v^{2}}\left[\frac{116 H^{3}}{35 b} i_{y}+\frac{32 b^{3}}{105 H} i_{z}+\frac{8 H b}{75} v\left(i_{y}+i_{z}\right)\right. \\
& \left.+\frac{143 H b}{75}(1-v) i_{t}\right] E \psi^{\prime 2}
\end{aligned}
$$

where $i_{y}, i_{z}$ and $i_{t}$ are the average moments of inertia of cross-section and torsional modulus per unit breadth, respectively. The stool strain energy is comprised of the bending, shear and torsional contributions

$U_{s}=\left[\frac{12 h^{2} I_{s b}}{b}+72(1+v) \frac{h^{2}}{b^{3}} \frac{I_{s b}^{2}}{A_{s}}+\frac{9 b I_{s t}}{10(1+v)}\right] E \psi^{\prime 2}$

where $I_{s b}, A_{s}$ and $I_{s t}$ are the moment of inertia of cross-section, shear area and torsional modulus, respectively. Quantity $h$ is the stool distance from the inner bottom, Figure 3.

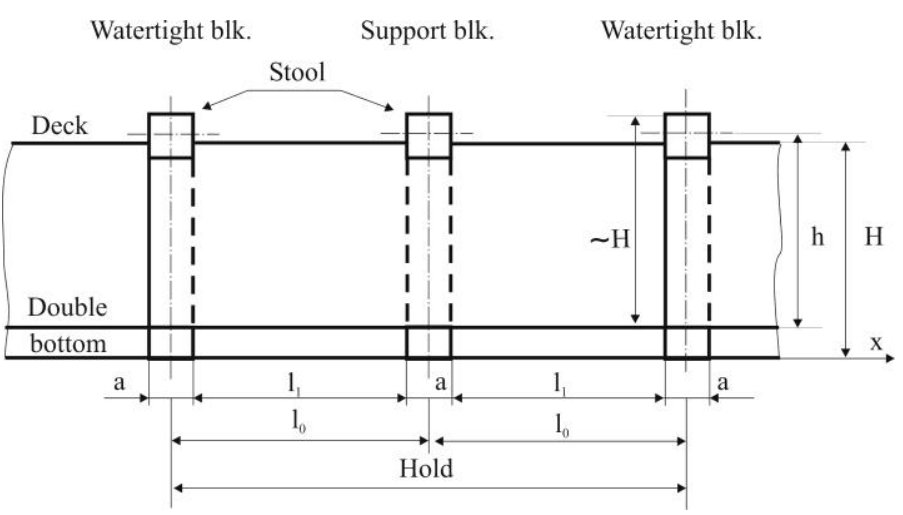

Figure 3. Longitudinal section of container ship hold

The equivalent torsional modulus yields, Figure 3

$I_{t}^{*}=\left[1+\frac{a}{l_{1}}+\frac{4(1+v) C}{I_{t} l_{0}}\right] I_{t}, \quad C=\frac{U_{g}+U_{s}}{E \psi^{\prime 2}}$,

where $a$ is the web height of bulkhead girders (frame spacing), $l_{0}$ is the bulkhead spacing, $l_{1}=l_{0}-a$ is the net length, and $C$ is the energy coefficient.

\subsection{Natural vibration analysis}

If the FEM approach is used, the governing equation of dry natural vibrations yields [18]

$\left(\mathbf{K}-\Omega^{2} \mathbf{M}\right) \boldsymbol{\delta}=\mathbf{0}$,

where $\mathbf{K}$ is stiffness matrix, $\mathbf{M}$ is mass matrix, $\Omega$ is dry natural frequency and $\boldsymbol{\delta}$ is dry natural mode. As solution of the eigenvalue problem (13) $\Omega_{i}$ and $\boldsymbol{\delta}_{i}$ are obtained for each the $i$-th dry mode, where $i=1,2 \ldots N, N$ is total number of degrees of freedom. Now natural modes matrix can be constituted

$$
\boldsymbol{\delta}=\left[\boldsymbol{\delta}_{1}, \boldsymbol{\delta}_{2} \ldots \boldsymbol{\delta}_{i} \ldots \boldsymbol{\delta}_{N}\right]
$$

and the modal stiffness and mass can be determined [19]
$\mathbf{k}=\boldsymbol{\delta}^{T} \mathbf{K} \boldsymbol{\delta}, \quad \mathbf{m}=\boldsymbol{\delta}^{T} \mathbf{M} \boldsymbol{\delta}$.

Generally the first six natural frequencies $\Omega_{i}$ are zero with corresponding eigenvectors representing the rigid body modes. As a result, the first six diagonal elements of $\mathbf{k}$ are also zero, while the first three elements in $\mathbf{m}$ are equal to structure mass, and the next three elements represent the mass moment of inertia around the corresponding coordinate axes.

If $1 \mathrm{D}$ analysis is applied, the beam modes are spread to the ship wetted surface using the expressions for vertical vibrations [2]

$\mathbf{h}_{i}=-\frac{\mathrm{d} w_{v i}}{\mathrm{~d} x}\left(z-z_{N}\right) \mathbf{i}+w_{v i} \mathbf{k}$,

and for coupled horizontal and torsional vibrations

$\mathbf{h}_{i}=\left(-\frac{\mathrm{d} w_{h i}}{\mathrm{~d} x} y+\frac{\mathrm{d} \psi_{i}}{\mathrm{~d} x} \bar{u}\right) \mathbf{i}+\left[w_{h i}+\psi_{i}\left(z-z_{S}\right)\right] \mathbf{j}-\psi_{i} y \mathbf{k}$,

where $w$ is hull deflection, $\psi$ is twist angle, $y$ and $z$ are coordinates of the point on ship surface, and $z_{N}$ and $z_{S}$ are coordinates of centroid and shear centre respectively, and $\bar{u}=\bar{u}(x, y, z)$ is the cross-section warping intensity reduced to the wetted surface [20].

\section{CONTRIBUTION OF ENGINE ROOM STRUCTURE TO HULL STIFFNESS}

Ultra Large Container Ships are characterized by relatively short engine room structure with length of about a half of ship breadth. Its complex deformation is illustrated in a case of a 7800 TEU container ship, Figure 4. The deck shear deformation is predominant, while hold transverse bulkhead stool is exposed to bending. Due to shortness of the engine room, its transverse bulkheads are skewed but somewhat less pronounced than warping of the hold bulkheads. Warping of the transom is negligible, and that is an important fact when specifying boundary conditions in vibration analysis.

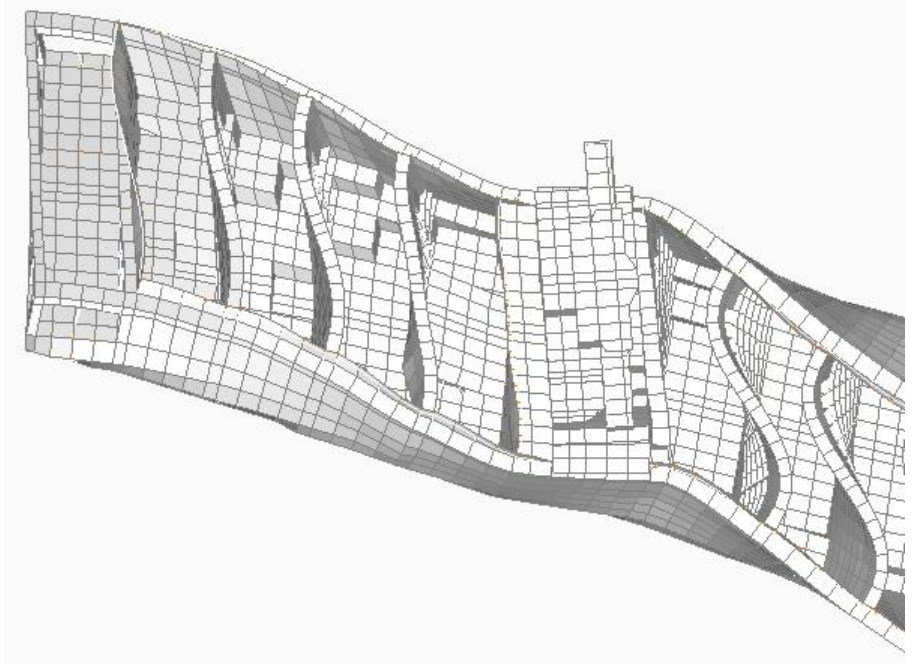

Figure 4. Deformation of 7800 TEU container ship aft structure 


\subsection{Stiffness of engine room structure}

A short engine room structure can be considered either as a closed segment with relevant stiffness or as an open segment with increased stiffness due to deck contribution [21]. The latter simulation in fact gives results which agree better with 3D FEM results, than the former one [22]. Deck contribution to hull stiffness can be determined by energy approach, as it is done in the case of transverse bulkheads [11]. Such a beam model is consistent at global level of energy balance, and that is sufficient for application in ship hydroelastic analysis, where proper natural frequencies and mode shapes of dry hull are required.

In the case of short engine room, torsion induces distortion of cross-section while hull bending is negligible. Solution of that complex problem is described here by employing the energy balance approach and concept of the effective stiffness due to reason of simplicity. A closed hull segment is considered as open one with deck influence. For that purpose let us determine deck strain energy. All quantities related to closed and open cross-section are designated by $(.)^{*}$ and $(.)^{\circ}$, respectively

As it can be seen in Figure 4, the upper deck is exposed to large deformation, while the double bottom in-plane deformation is quite small. The relative axial displacement of the internal upper deck boundaries, with respect to double bottom, is result of their warping

$$
U=\left|U_{D}\right|+\left|U_{B}\right|=\left(\left|w_{D}\right|+\left|w_{B}\right|\right) \psi_{t}^{\prime}
$$

It causes deck in-plane (membrane) deformation. The problem can be solved in an approximate analytical way by considering deck as a beam. Its horizontal anti-symmetric deflection consists of pure bending and shear contribution, Figure 5. The former is assumed in the form

$u_{b}=\frac{y}{2 b}\left[3-\left(\frac{y}{b}\right)^{2}\right] U_{b}$,

which satisfies relevant boundary conditions: $u_{b}(0)=0$ and $u_{b}^{\prime \prime}(0)=0$, where $U_{b}$ is the boundary bending deflection. Shear deflection depends on bending deflection

$u_{s}=-\frac{E I}{G A} \frac{\mathrm{d}^{2} u_{b}}{\mathrm{~d} y^{2}}=2(1+v)\left(\frac{a}{b}\right)^{2} \frac{y}{b} U_{b}$,

where the internal deck cross-section area, $A=2 a t$, its moment of inertia, $I=\frac{2}{3} a^{3} t$, and the relation $E=2(1+v) G$, are taken into account, Figure 5. Total deflection is obtained by summing up its constitutive parts, Eqs. (19) and (20). Relation between total boundary deflection and the bending boundary deflection reads

$U=\left[1+2(1+v)\left(\frac{a}{b}\right)^{2}\right] U_{b}$

The total internal deck strain energy consists of the bending and shear contributions

$E_{1}=\frac{1}{2} E I \int_{-b}^{b}\left(\frac{\mathrm{d}^{2} u_{b}}{\mathrm{~d} y^{2}}\right)^{2} \mathrm{~d} y+\frac{1}{2} G A \int_{-b}^{b}\left(\frac{\mathrm{d} u_{s}}{\mathrm{~d} y}\right)^{2} \mathrm{~d} y$
By substituting Eqs. (19) and (20) into (22), one finds

$$
E_{1}=\frac{1}{2} E I \int_{-b}^{b}\left(\frac{\mathrm{d}^{2} u_{b}}{\mathrm{~d} y^{2}}\right)^{2} \mathrm{~d} y+\frac{1}{2} G A \int_{-b}^{b}\left(\frac{\mathrm{d} u_{s}}{\mathrm{~d} y}\right)^{2} \mathrm{~d} y
$$
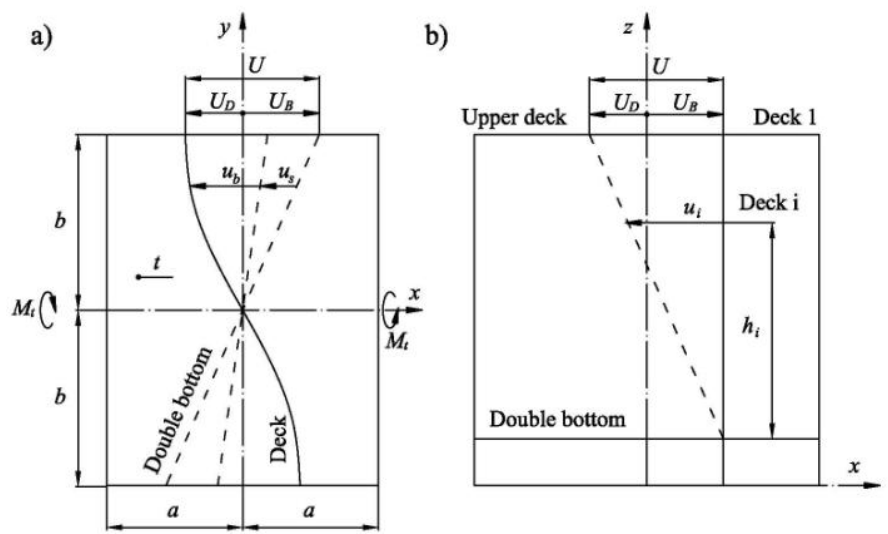

\section{Figure 5. Deck deformation and double bottom rotation, a)-bird view, b)-lateral view}

Finally, by taking into account Eqs. (18) and (21), yields

$$
E_{1}=\frac{4(1+v) G t\left(\frac{a}{b}\right)^{3}}{1+2(1+v)\left(\frac{a}{b}\right)^{2}}\left(\left|w_{D}\right|+\left|w_{B}\right|\right)^{2} \psi_{t}^{\prime 2}
$$

On the other hand, total energy of the closed hull segment can be obtained by summing up energy of open segment and the deck strain energy, i.e.

$E_{\text {tot }}^{*}=E_{w}^{\circ}+E_{t}^{\circ}+E_{1}-E_{\mu}$

where

$$
E_{w}^{\circ}=\frac{1}{2} \int_{-a}^{a} B_{w}^{\circ} \psi_{t}^{\prime \prime} \mathrm{d} x, \quad E_{t}^{\circ}=\frac{1}{2} \int_{-a}^{a} T_{t}^{\circ} \psi_{t}^{\prime} \mathrm{d} x, \quad E_{\mu}=\int_{-a}^{a} \mu_{x} \psi \mathrm{d} x .
$$

Within a short span $2 a$, constant value of $\psi_{t}^{\prime}$ (as for deck) can be assumed, so that second term in Eq. (26) by inserting $T_{t}^{\circ}$ from Eq. (7), leads to

$E_{t}^{\circ}=G I_{t}^{\circ} a \psi_{t}^{\prime 2}$.

$E_{t}^{\circ}$ and $E_{1}$ in (25) can be unified into one term since both depend on $\psi_{t}^{\prime 2}$

$E_{t}^{\circ}+E_{1}=G a \tilde{I}_{t} \psi_{t}^{\prime 2}$

where

$\tilde{I}_{t}=(1+C) I_{t}^{\circ}, \quad C=\frac{E_{1}}{E_{t}^{\circ}}$ 
$\tilde{I}_{t}$ is the effective torsional modulus which includes both open crosssection and deck effects.

Engine room structure is designed in such a way that the hold double skin continuity is ensured and necessary decks are inserted between the double skins. Strain energy is derived for the first (main) deck and for the others it can be assumed that their strain energy is proportional to the deck plating volume, $V$, and linearly increasing deformation with the deck distance from inner bottom, $h$, Figure 5, since the double bottom is much stiffer than decks. In that way the coefficient $C$, Eq. (29b), by employing (24) and (27), reads

$C=\frac{\sum E_{i}}{E_{t}}=\frac{4(1+v) t_{1}\left(\frac{a}{b}\right)^{3}\left(\left|w_{D}\right|+\left|w_{B}\right|\right)^{2} k}{\left[1+2(1+v)\left(\frac{a}{b}\right)^{2}\right] I_{t}^{\circ} a}$

where

$k=\sum \frac{V_{i}}{V_{1}}\left(\frac{h_{i}}{h_{1}}\right)^{2}$.

In the above consideration distortion of cross-sections is not included and that is subject of further investigation.

\subsection{Torsion of segmented girder}

Couple of problems are related to the beam modelling of container ship structures: connection of closed parts, i.e. fore and aft peaks and engine room, with open holds and accounting for transverse bulkheads effect. Due to different vertical position of the shear centre, coupling between torsion and horizontal bending exists within displacements and sectional forces; $Y^{*}=Y^{\circ}+\psi\left(z_{S C}^{*}-z_{S C}^{\circ}\right), \quad T^{\circ}=T^{*}+Q\left(z_{S C}^{*}-z_{S C}^{\circ}\right)$

where $Y$ is deflection, $\psi$ is twist angle, $T$ is torque and $Q$ is shear force, $z_{S C}$ is coordinate of shear centre. Warping compatibility in the joint of open and closed cross-section presents another problem which can be solved in the conventional or an advanced way, respectively:

a) Equilibrium of bimoments, $B_{w}$, and compatibility of twist angle derivatives, $\psi^{\prime}$. Coupling between torsion and bending on the compatibility basis is avoided.

b) Discontinuity of twist angle derivatives $\psi^{\prime}\left(x^{+}\right)=s_{1} \psi^{\prime}\left(x^{-}\right)$, and coupling between bending angles and twist angle $\varphi\left(x^{+}\right)=\varphi\left(x^{-}\right)+s_{2} \psi^{\prime}\left(x^{-}\right) ; \quad$ equilibrium of bending moments $\quad M\left(x^{-}\right)=M\left(x^{+}\right) \quad$ and bimoments $B_{w}\left(x^{-}\right)=s_{1} B_{w}\left(x^{+}\right)-s_{2} M(x)$, where $s_{1}$ and $s_{2}$ are the warping compatibility factors which depend on warping function, [22, 23].

The girder consisted of three segments is under consideration, Figure 6 . The end segments are open and the middle one is closed, so that the girder is symmetric with respect to the $z$ axis. Each segment is specified in its local coordinate system. The relevant expressions for displacements and sectional forces are listed below [8]:

$$
\begin{aligned}
& u=w \frac{\mathrm{d} \psi_{t}}{\mathrm{~d} x}=w\left(\frac{A_{1}}{l}+A_{2} \alpha \operatorname{sh} \alpha x+A_{3} \alpha \operatorname{ch} \alpha x+\psi_{p}^{\prime}\right), \\
& T_{t}=G I_{t}\left(\frac{A_{1}}{l}+A_{2} \alpha \operatorname{sh} \alpha x+A_{3} \alpha \operatorname{ch} \alpha x+\psi_{p}^{\prime}\right), \\
& T_{w}=-G I_{t}\left(A_{2} \alpha \operatorname{sh} \alpha x+A_{3} \alpha \operatorname{ch} \alpha x\right)-E I_{w} \psi_{p}^{\prime \prime \prime}, \\
& T=G I_{t}\left(\frac{A_{1}}{l}+\psi_{p}^{\prime}\right)-E I_{w} \psi_{p}^{\prime \prime \prime}, \\
& B_{w}=G I_{t}\left(A_{2} \operatorname{ch} \alpha x+A_{3} \operatorname{sh} \alpha x\right)+E I_{w} \psi_{p}^{\prime \prime},
\end{aligned}
$$

where $\psi_{p}$ represents particular solution of differential equation and coefficient $\alpha$ yields

$$
\alpha=\sqrt{\frac{G I_{t}}{E I_{w}}} .
$$

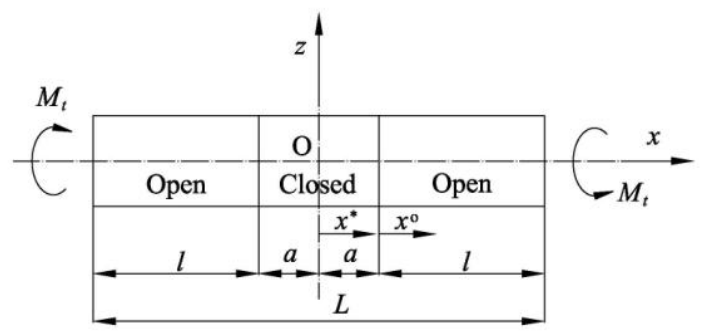

Figure 6. Torsion of segmented girder

Symbols $A_{i}$ and $B_{i}$ are used for integration constants of closed and open segment.

Compatibility coefficients $s_{1}$ and $s_{2}$ in the formulation (a) of compatibility conditions, depends on $I_{w}^{*}$ and $I_{w}^{\circ}$ [22]. Since $I_{w}^{\circ}$ instead of $I_{w}^{*}$ is taken into account, $s_{1}=1$ and $s_{2}=0$. As a result, the torsion doesn't induce horizontal bending so that the conventional compatibility conditions (a) at the joint of closed and open segments can be used. Thus, the boundary and compatibility conditions in the considered case, yield

$\psi^{*}(a)=\psi^{\circ}(0), \psi_{t}^{* \prime}(a)=\psi_{t}^{\circ}(0)$,

$T^{*}(a)=T^{\circ}(0), B_{w}^{*}(a)=B_{w}^{\circ}(0)$,

$u^{\circ}\left(l^{\circ}\right)=0, T^{\circ}\left(l^{\circ}\right)=M_{t}$.

From the third and last conditions (34) one finds

$$
A_{1}=\frac{M_{t} a}{G \tilde{I}_{t}}, B_{1}=\frac{M_{t} l^{\circ}}{G I_{t}^{\circ}}
$$

The remaining four conditions (34) lead to the following system of algebraic equations:

$$
\left[\begin{array}{ccc}
\alpha \operatorname{ch} \alpha a & 0 & -\beta \\
\tilde{I} \operatorname{sh} \alpha a & -I_{t}^{\circ} & 0 \\
0 & \beta \operatorname{sh} \beta l^{\circ} & \beta \operatorname{ch} \beta l^{\circ}
\end{array}\right]\left\{\begin{array}{l}
A_{3} \\
B_{2} \\
B_{3}
\end{array}\right\}=\frac{M_{t}}{G}\left\{\begin{array}{c}
\frac{1}{I_{t}^{\circ}}-\frac{1}{\tilde{I}_{t}} \\
0 \\
-\frac{1}{I_{t}^{\circ}}
\end{array}\right\}
$$


$B_{0}=A_{1}+A_{3}\left(1-\frac{\tilde{I}_{t}}{I_{s}^{\circ}}\right) \operatorname{sh} \alpha a-B_{2}\left(1-\frac{I_{t}^{\circ}}{I_{s}^{\circ}}\right)$,

where

$\alpha=\sqrt{\frac{G \tilde{I}_{t}}{E I_{w}^{\circ}}}, \beta=\sqrt{\frac{G I_{t}^{\circ}}{E I_{w}^{\circ}}}$.

The solution of system (36) reads

$A_{3}=\frac{D_{A 3}}{D}, B_{2}=\frac{D_{B 2}}{D}, B_{3}=\frac{D_{B 3}}{D}$,

where

$$
\begin{aligned}
& D_{A 3}=\frac{M_{t}}{G}\left[\left(1-\frac{I_{t}^{\circ}}{\tilde{I}_{t}}\right) \operatorname{ch} \beta l^{\circ}-1\right], \\
& D_{B 2}=-\frac{M_{t}}{G} \operatorname{sh} \alpha a\left[\left(1-\frac{\tilde{I}_{t}}{I_{t}^{\circ}}\right) \operatorname{ch} \beta l^{\circ}+\frac{\tilde{I}_{t}}{I_{t}^{\circ}}\right], \\
& D_{B 3}=\frac{M_{t}}{G}\left[\left(1-\frac{\tilde{I}_{t}}{I_{t}^{\circ}}\right) \operatorname{sh} \alpha a \operatorname{sh} \beta l^{\circ}-\frac{\alpha}{\beta} \operatorname{ch} \alpha a\right], \\
& D=I_{t}^{\circ} \alpha \operatorname{ch} \alpha a \operatorname{ch} \beta l^{\circ}+\tilde{I}_{t} \beta \operatorname{sh} \alpha a \operatorname{sh} \beta l^{\circ} .
\end{aligned}
$$

\section{HYDRODINAMIC MODEL}

Harmonic hydroelastic problem is considered in frequency domain and therefore one operates with amplitudes of forces and displacements. In order to perform structural and hydrodynamic coupling, it is useful to split total hydrodynamic force $F^{h}$ into two parts: the first part $F^{R}$ depending on the structural deformations, and the second one $F^{D I}$ representing the pure excitation [13]. Furthermore, the modal superposition method is used. Vector of the wetted surface deformations $\mathbf{H}(x, y, z)$ can be presented as a series of dry natural modes $\mathbf{h}_{i}(x, y, z)$.

The potential theory assumptions are adopted for the hydrodynamic part of the problem and the total velocity potential $\varphi$, in the case of no forward speed, is defined with the Laplace differential equation and the given boundary values. Furthermore, the linear wave theory enables decomposition of the total potential [24]

$\varphi=\varphi_{I}+\varphi_{D}-i \omega \sum_{j=1}^{N} \xi_{j} \varphi_{R j}, \quad \varphi_{I}=-i \frac{g A}{\omega} \mathrm{e}^{\nu(z+i x)}$,

where $\varphi_{I}$ is incident wave potential, $\varphi_{D}$ is diffraction potential, $\varphi_{R j}$ is radiation potential and $A$ and $\omega$ represent wave amplitude and frequency respectively. Once the potentials are determined, the modal hydrodynamic forces are calculated by pressure work integration over the wetted surface, $S$. The total linearised pressure can be found from Bernoulli's equation

$p=i \omega \rho \varphi-\rho g z$.

First, the term associated with the velocity potential $\varphi$ is considered and subdivided into excitation and radiation parts

$F_{i}^{D I}=i \omega \rho \iint_{S}\left(\varphi_{I}+\varphi_{D}\right) \mathbf{h}_{i} \mathbf{n d} S$,
$F_{i}^{R}=\rho \omega^{2} \sum_{j=1}^{N} \xi_{j} \iint_{S} \varphi_{R j} \mathbf{h} \mathbf{n} \mathrm{d} S$

Thus, $F_{i}{ }^{D I}$ represents the modal pressure excitation. Now, one can decompose (44) into the modal inertia force and damping force associated with acceleration and velocity, respectively

$F_{i}^{a}=\operatorname{Re}\left(F_{i}^{R}\right)=\omega^{2} \sum_{j=1}^{N} \xi_{j} A_{i j}, A_{i j}=\rho \operatorname{Re} \iint_{S} \varphi_{R j} \mathbf{h}_{i} \mathbf{n d} S$,

$F_{i}^{v}=\operatorname{Im}\left(F_{i}^{R}\right)=\omega \sum_{j=1}^{N} \xi_{j} B_{i j}, \quad B_{i j}=\rho \omega \operatorname{Im} \iint_{S} \varphi_{R j} \mathbf{h}_{i} \mathbf{n} \mathrm{d} S$.

where $A_{i j}$ and $B_{i j}$ are elements of added mass and damping matrices, respectively.

Determination of added mass and damping for rigid body modes is a well-known procedure in ship hydrodynamics. Now the same procedure is extended to the calculation of these quantities for elastic modes. The hydrostatic part of the total pressure, $-\rho g z$ in (42), is considered within the hydrostatic model.

\section{HYDROSTATIC MODEL}

There are few solutions for restoring stiffness in the literature [24, 25, $26,27]$. In this study consistent formulation of restoring stiffness for ships is used [2, 7], and here only basic formulae are given.

The restoring stiffness consists of hydrostatic and gravity parts. Work of the hydrostatic pressure as the generalized force can be written in the form

$$
F^{h}=-\rho g \iint_{S}\left[H_{z}+Z(\nabla \mathbf{H})\right] \mathbf{H n d} S,
$$

where $\nabla$ is Hamilton differential operator, $\mathbf{H}$ is displacement vector, $\mathrm{d} S$ is differential of wetted surface, $Z$ is its depth and $\mathbf{n}$ is unit normal vector. Stiffness is generally defined as a relation between incremental force and displacement, so it is determined from the variational equation

$\delta F^{h}=-\rho g \iint_{S}\left[H_{z}+Z(\nabla \mathbf{H})\right] \delta \mathbf{H n d} S$.

Furthermore, the modal superposition method is used, and the variation is transmitted to modes, i.e. modal forces and displacements

$$
\delta F^{h}=\sum_{j=1}^{N} \delta F_{j}^{h}, \quad \mathbf{H}=\sum_{j=1}^{N} \xi_{j} \mathbf{h}_{j}, \quad \delta \mathbf{H}=\sum_{j=1}^{N} \mathbf{h}_{j} \delta \xi_{j} .
$$

In that way, Eq. (48) is decomposed into the modal equations

$\delta F_{i}^{h}=-\sum_{j=1}^{N}\left[\left(C_{i j}^{p}+C_{i j}^{n h}\right) \xi_{j}\right] \delta \xi_{i}$,

where

$C_{i j}^{p}=\rho g \iint_{S} \mathbf{h}_{i} h_{z}^{j} \mathbf{n d} S, \quad C_{i j}^{n h}=\rho g \iint_{S} Z \mathbf{h}_{i}\left(\nabla \mathbf{h}_{j}\right) \mathbf{n} \mathrm{d} S$,

are stiffness coefficients due to pressure, and normal vector and mode contributions, respectively.

Similarly to the pressure part, the generalized gravity force reads 


$$
F^{m}=-g \iiint_{V} \rho_{s}(\mathbf{H} \nabla) H_{z} \mathrm{~d} V,
$$

where $\rho_{s}$ and $V$ are structure density and volume, respectively. By obtaining consistent variational equation and then by applying modal superposition method similarly as for hydrostatic part, the following modal variational equation is obtained

$\delta F_{i}^{m}=-\sum_{j=1}^{N} C_{i j}^{m} \xi_{j} \delta \xi_{i}$,

where

$C_{i j}^{m}=g \iiint_{V} \rho_{s}\left(\mathbf{h}_{i} \nabla\right) h_{z}^{j} \mathrm{~d} V$,

are the gravity stiffness coefficients. Complete restoring stiffness coefficients are obtained by summing up its constitutive parts

$C_{i j}=C_{i j}^{p}+C_{i j}^{n h}+C_{i j}^{m}$.

The geometric stiffness, which takes initial stresses at the calm sea into account, is not considered here, since its contribution to the restoring stiffness is quite small for ship structures [27].

\section{HYDROELASTIC MODEL}

After the definition of the structural, hydrostatic and hydrodynamic models, the hydroelastic model can be constituted. The governing matrix differential equation for coupled ship motions and vibrations is deduced

$\left[\mathbf{k}+\mathbf{C}-i \omega(\mathbf{d}+\mathbf{B}(\omega))-\omega^{2}(\mathbf{m}+\mathbf{A}(\omega))\right] \boldsymbol{\xi}=\mathbf{F}$,

where $\mathbf{k}, \mathbf{d}$, and $\mathbf{m}$ are structural stiffness, damping and mass matrices, respectively, $\mathbf{C}$ is restoring stiffness, $\mathbf{B}(\omega)$ is hydrodynamic damping, $\mathbf{A}(\omega)$ is added mass, $\boldsymbol{\xi}$ is modal amplitudes, $\mathbf{F}$ is wave excitation and $\omega$ is encounter frequency. All quantities, except $\omega$ and $\xi$, are related to the dry modes. The solution of (56) gives the modal amplitudes $\xi_{i}$ and displacement of any point of the structure obtained by re-tracking to (49).

\section{NUMERICAL EXAMPLES}

For the illustration of the procedure related to engine room effective stiffness determination, 3D FEM analysis of ship-like pontoon has been undertaken. The 3D FEM model is constituted according to 7800 TEU container ship with main dimensions $L_{p p} \times B \times H=319 \times 42.8 \times 24.6 \mathrm{~m}$, Figure 7, [9]. The complete hydroelastic analysis of the same ship has been performed.

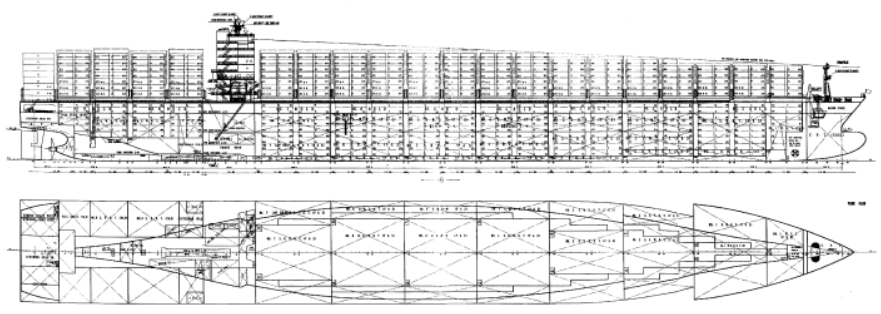

Figure 7. 7800 TEU Container Ship
Stiffness properties of ship hull are calculated by program STIFF, based on the theory of thin-walled girders [28], Figure 8.

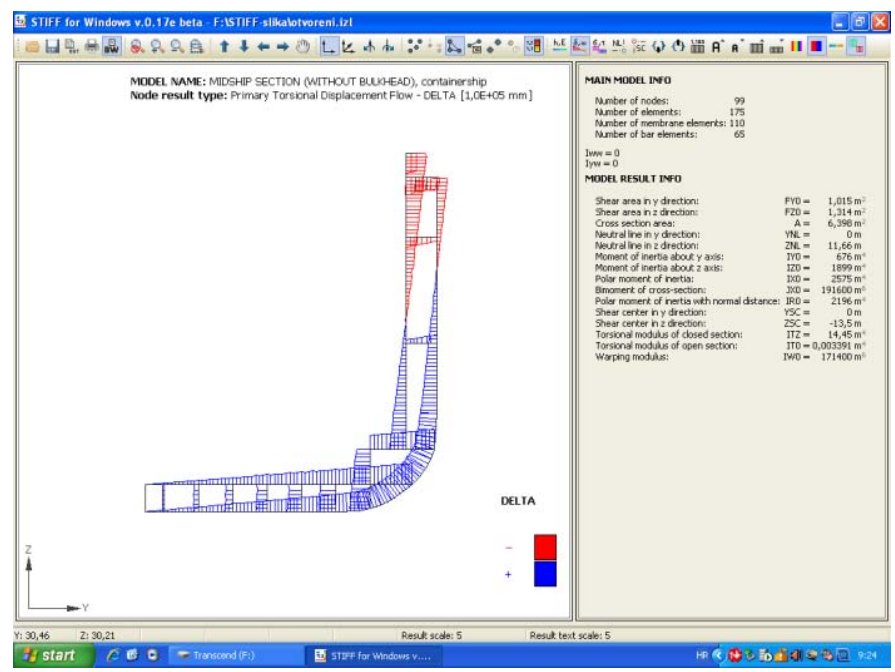

Figure 8. Program STIFF - warping of ship cross-section

Influence of the transverse bulkheads is taken into account by using the equivalent torsional modulus for the open cross-sections instead of the actual values, i.e. $I_{t}^{*}=2.4 I_{t}$. This value is applied for all shipcross sections as the first approximation.

\subsection{Analysis of ship-like segmented pontoon}

Torsion of the segmented pontoon of the length $L=300 \mathrm{~m}$, with effective parameters is considered. Torsional moment $M_{t}=40570$ $\mathrm{kNm}$ is imposed at the pontoon ends. The pontoon is considered free in the space and the problem is solved analytically according to the formulae given in Section 4. The following values of the basic parameters are used: $a=10.1 \mathrm{~m}, b=19.17 \mathrm{~m}, t_{1}=0.01645 \mathrm{~m}$, $w_{D}=-221 \mathrm{~m}^{2}, w_{B}=267 \mathrm{~m}^{2}, I_{t}^{\circ}=14.45 \mathrm{~m}^{4}, k=1.894$. As a result $C=22.42$, Eq. (30), and accordingly $\tilde{I}_{t}=338.4 \mathrm{~m}^{4}$, Eq. (29a), are obtained. Since $\tilde{I}_{t}=0.36 I_{t}^{*}$, effect of the short engine room structure on its torsional stiffness is obvious.

The 3D FEM model of segmented pontoon is made by commercial software package SESAM and consists of 20 open and 1 closed (engine room) superelement. The pontoon ends are closed with transverse bulkheads. The shell finite elements are used. The pontoons are loaded at their ends with the vertical distributed forces in the opposite directions, generating total torque $M_{t}=40570 \mathrm{kNm}$. The midship section is fixed against transverse and vertical displacements, and the pontoon ends are constrained against axial displacements (warping). Lateral and bird view on the deformed segmented pontoon is shown in Figure 9, where the influence of more rigid engine room structure is evident. Detailed view on this pontoon portion is presented in Figure 10. It is apparent that segment of very stiff double bottom and sides rotate as a "rigid body", while decks and transverse bulkheads are exposed to shear deformation. This deformation causes the distortion of the cross-section, Figure 10. 

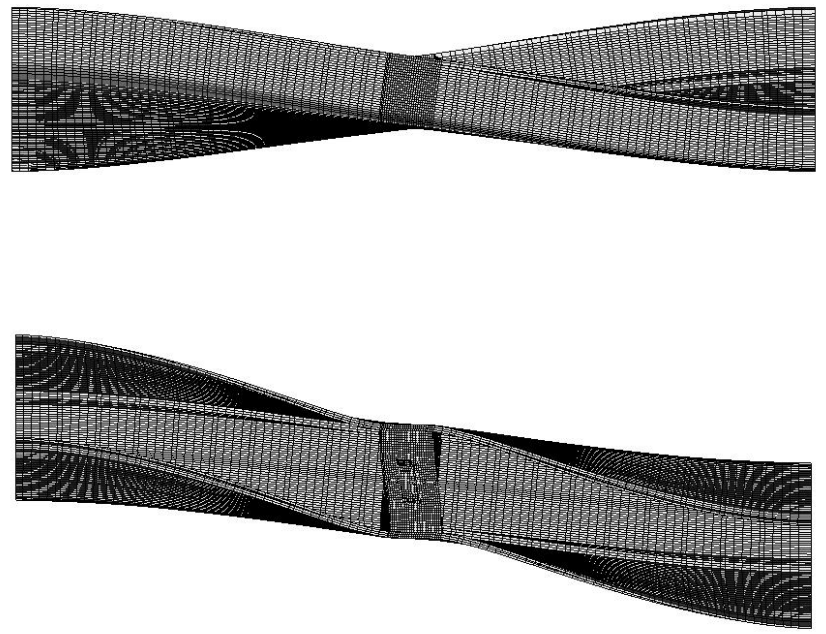

Figure 9. Deformation of segmented pontoon, lateral and bird view
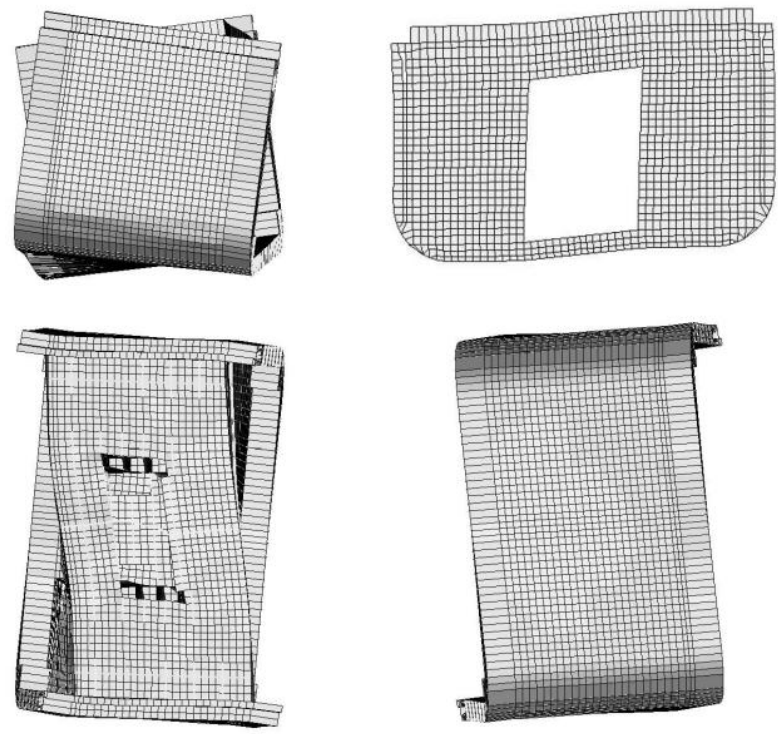

Figure 10. Lateral, axial, bird and fish views on deformed engine room superelement

Twist angles of the analytical beam solution and that of 3D FEM analysis for the pontoon bottom are compared in Figure 11. As it can be noticed, there are some small discrepancies between $\psi_{(1+2) D}$ and $\psi_{3 D, b o t t o m}$, which are reduced to a negligible value at the pontoon ends

Figure 11 also shows twist angle of side structure and the difference $\delta=\psi_{3 \mathrm{D} \text {,bottom }}-\psi_{3 \mathrm{D} \text {, side }}$ represents distortion angle of cross-section which is highly pronounced. As it is mentioned before, the problem will be further investigated.

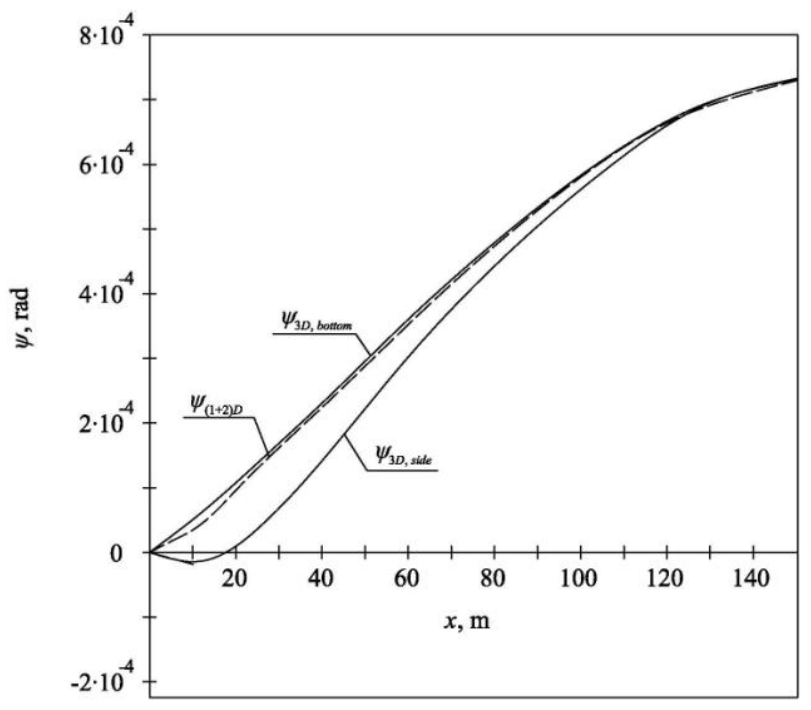

Figure 11. Twist angles of segmented pontoon

\subsection{Validation of 1D FEM model}

The reliability of 1D FEM analysis is verified by 3D FEM analysis of the considered ship. For this purpose, the light weight loading condition of dry ship with displacement $\Delta=33692 \mathrm{t}$ is taken into account. The equivalent torsional stiffness of the engine room structure, as well as equivalent stiffness of fore and aft peaks is not taken into account in this example for the time being. However, it will be done in the next step of investigation. The lateral and bird view of the first dominantly torsional mode of the wetted surface, determined by $1 \mathrm{D}$ model, is shown in Figure 12.

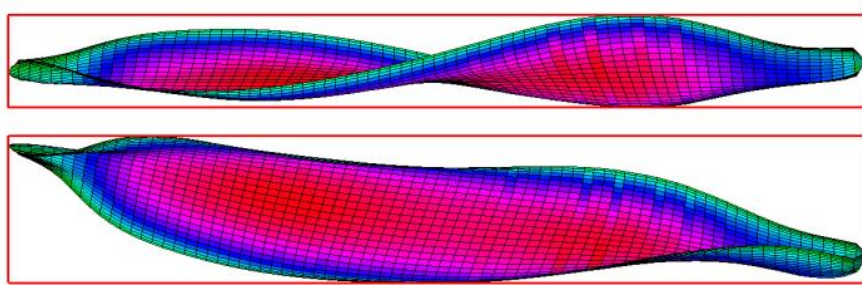

Figure 12. The first dominantly torsional mode, lateral and bird view, light weight, 1D model

The first 3D dry coupled natural modes of the complete ship structure is shown in Figure 13. It is similar to that of 1D analysis for the wetted surface. Warping of the transverse bulkheads, which increases the hull torsional stiffness, is evident.

The first four corresponding natural frequencies obtained by 1D and 3D analyses are compared in Table 1.

Table 1. Dry natural frequencies, light weight, $\omega_{i}[\mathrm{rad} / \mathrm{s}]$

\begin{tabular}{cccccc}
\hline \multirow{2}{*}{$\begin{array}{c}\text { Mode } \\
\text { no. }\end{array}$} & \multicolumn{2}{c}{ Vert. } & \multicolumn{2}{c}{ Horiz. + tors. } & \multirow{2}{*}{ Mode no. } \\
\cline { 2 - 5 } & 1D & 3D & 1D & 3D & \\
\hline 1 & 7.35 & 7.33 & 4.17 & 4.15 & $1(\mathrm{H} 0+\mathrm{T} 1)$ \\
2 & 15.00 & 14.95 & 7.34 & 7.40 & $2(\mathrm{H} 1+\mathrm{T} 2)$ \\
3 & 24.04 & 22.99 & 12.22 & 12.09 & $3(\mathrm{H} 2+\mathrm{T} 3)$ \\
4 & 35.08 & 34.21 & 15.02 & 16.22 & $4(\mathrm{H} 3+\mathrm{T} 4)$ \\
\hline
\end{tabular}




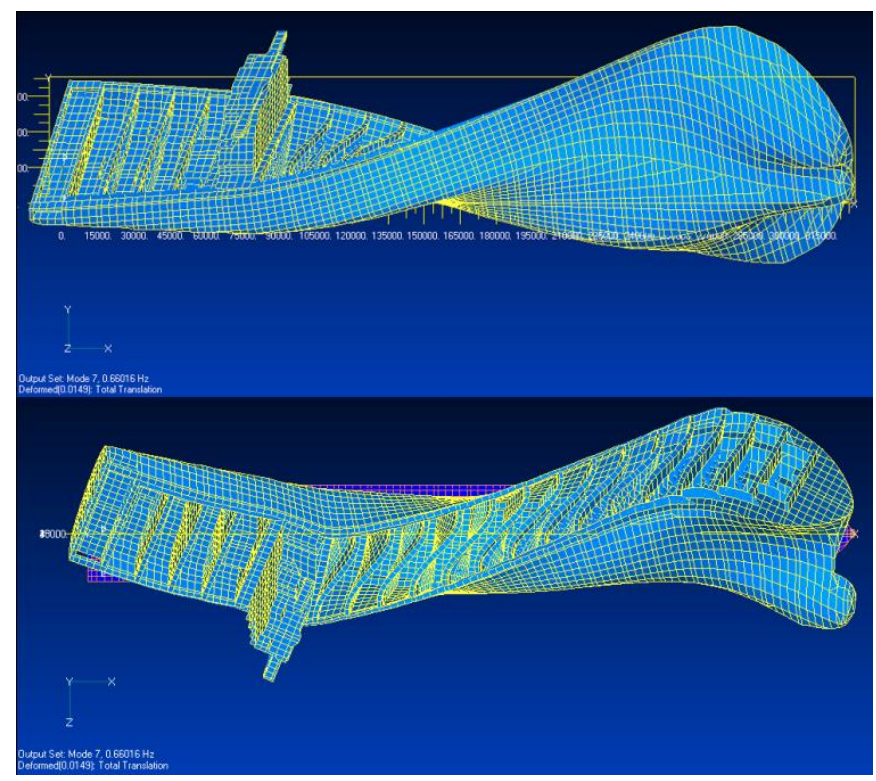

Figure 13. The first dominantly torsional mode, lateral and bird view, light weight, 3D model

Quite good agreement is achieved. Values of natural frequencies for higher modes are more difficult to correlate, since strong coupling between global hull modes and local substructure modes of 3D analysis occurs.

\subsection{Ship response}

Transfer functions of torsional moment and horizontal bending moment at the midship section, obtained using 1D structural model, are shown in Figures 14 and 15, respectively. The angle of $180^{\circ}$ is related to head sea. They are compared to the rigid body ones determined by program HYDROSTAR. Very good agreement is obtained in the lower frequency domain, where the ship behaves as a rigid body, while large discrepancies occur at the resonances of the elastic modes, as expected.

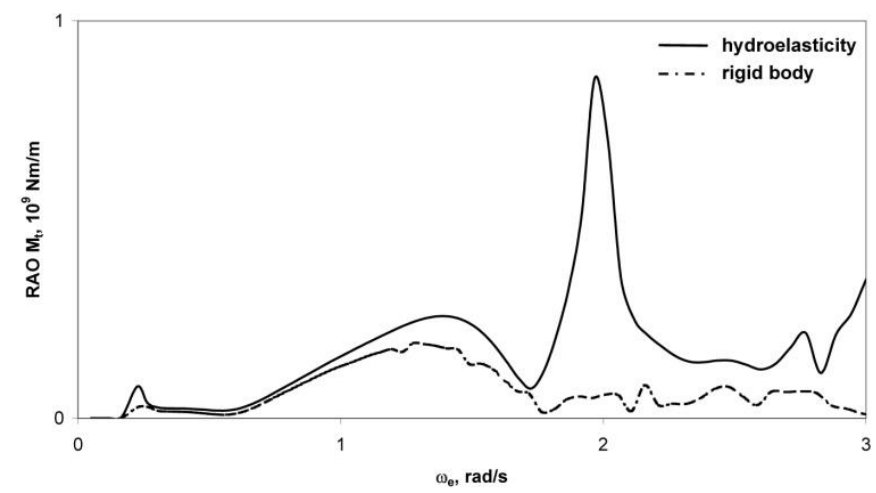

Figure 14. Transfer function of torsional moment, $X=120^{\circ}$, $U=25 \mathrm{kn}, x=155.75 \mathrm{~m}$ from AP

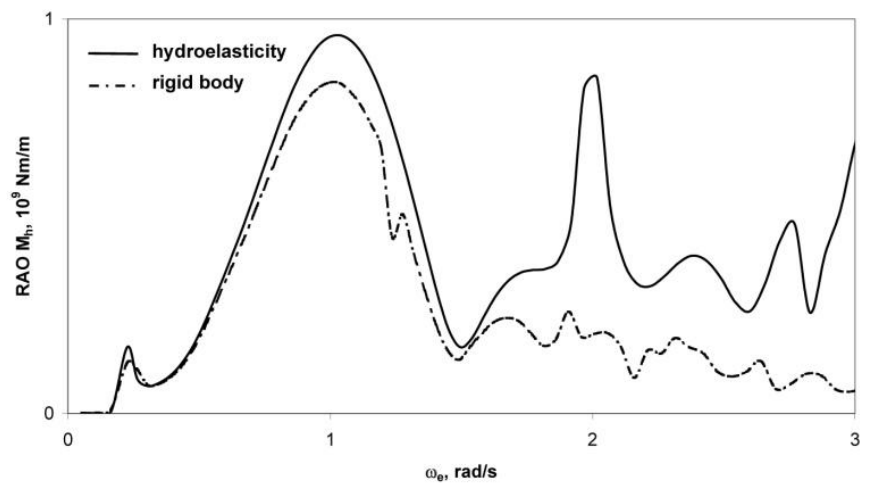

Figure 15. Transfer function of horizontal bending moment, $X=120^{\circ}, U=25 \mathrm{kn}, X=155.75 \mathrm{~m}$ from AP

Necessary condition for convergence of sectional forces to zero value as the wave frequency approaches to zero can be used as a benchmark for validation of the restoring stiffness. Figure 16 shows the zoomed transfer function of torsional moment determined by the direct integration and three formulations of restoring stiffness in the hydroelastic approach: consistent one from this paper, symmetric matrix obtained by the minimum energy method, and hybrid matrix [24]. Only the consistent restoring stiffness satisfies the above condition as the rigid body solution does. In the case of symmetric and hybrid matrices the ship is not equilibrated. Moreover, the consistent restoring stiffness emphasizes the roll resonance at $0.23 \mathrm{rad} / \mathrm{s}$.

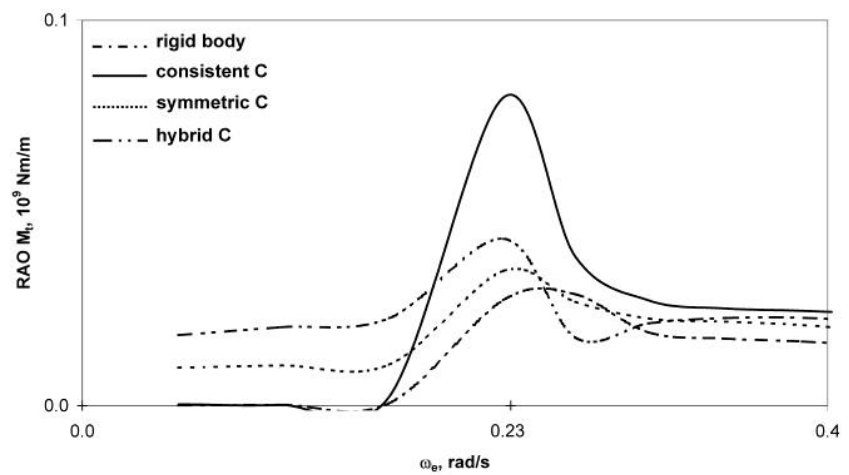

Figure 16. Zoomed transfer function of torsional moment, $X=120^{\circ}, U=25 \mathrm{kn}, X=155.75 \mathrm{~m}$ from AP

\section{CONCLUSIONS AND FUTURE WORK}

The illustrative numerical example of the 7800 TEU container ship shows that the developed hydroelasticity theory, utilizing sophisticated 1D FEM structural model and 3D hydrodynamic model, is an efficient tool for application in ship hydroelastic analyses. The obtained results point out that the transfer functions of hull sectional forces in case of resonant vibration (springing) are much higher than in resonant ship motion. Very good agreement between ship response determined by hydroelastic analysis and rigid body analysis in vicinity of zero frequency is obtained due to use of the consistent restoring stiffness. It should be mentioned that within the numerical example the structural damping has been neglected since its influence on the response is of second order. However, it can be taken into account as 2 or 3 percent of critical damping [9]. 
The used advanced beam model of ship hull, based on advanced thin-walled girder theory with included shear influence on torsion and a proper contribution of transverse bulkheads and engine room structure to its stiffness, is a reasonable choice for determining wave load effects. However, based on the experience, stress concentration in hatch corners calculated directly by the beam model is underestimated. This problem can be overcome by applying substructure approach, i.e. 3D FEM model of substructure with imposed boundary conditions from beam response. In any case, 3D FEM model of complete ship is preferable from the viewpoint of determining stress concentration. Concerning further improvements of the beam model, the distortion induced by torsion is of interest.

In order to complete hydroelastic analysis of container ships and confirm its importance for ship safety, it is necessary to proceed further to ship motion calculation in irregular waves for different sea states, based on the known transfer functions. This includes determination of global wave loads, i.e. bending and torsional moments and their conversion into stresses, stress concentration in critical areas of ship structures, especially in hatch corners due to restrained warping, and fatigue of structural details.

\section{ACKNOWLEDGMENTS}

This investigation is done within the EU FP7 Project Tools for Ultra Large Container Ships (TULCS) and the project Load and Response of Ship Structures funded by Croatian Ministry of Science, Education and Sports. The authors would like to express their gratitude to Dr. Stipe Tomašević for performing hydroelastic calculations of the considered ship.

\section{REFERENCES}

1. Salvesen, N., Tuck, EO., Faltinsen, OM. Ship motion and sea loads. Cambridge University Press, 1970.

2. Senjanović, I., Tomašević, S., Vladimir, N., Tomić, M., Malenica, Š. Ship hydroelastic analysis with sophisticated beam model and consistent restoring stiffness. Proc. Hydroelasticity in Marine Technology, University of Southampton, Southampton, 2009, p. 69-80.

3. Senjanović, I., Tomašević, S., Vladimir, N., Tomić, M., Malenica, $\underline{\text { S. Application of an advanced beam theory to ship hydroelastic }}$ analysis. Proc. Intl. Workshop on Advanced Ship Design for Pollution Prevention, Taylor \& Francis, London, 2010, p. 31-42.

4. Bishop, RED. \& Price WG. Hydroelasticity of Ships. Cambridge University Press, 1979.

5. Senjanović, I., Malenica, Š, Tomašević, S, Rudan, S. Methodology of ship hydroelastic investigation. Brodogradnja, 2007, 58(2), p. 133-145.

6. Senjanović, I., Tomašević, S., Tomić, M., Rudan, S., Vladimir, N., Malenica, Š. Hydroelasticity of Very Large Container Ships. Proc. Design and Operation Of Container Ships, RINA, London, 2008, p. 51-70.

7. Senjanović, I., Tomašević, S., Vladimir, N., Malenica, Š. Numerical procedure for ship hydroelastic analysis. Proc. Intl. Conf. Computational Methods in Marine Eng., CIMNE, Barcelona, 2009, p. 259-264.

8. Senjanović, I., Tomašević, S., Vladimir, N. An advanced theory of thin-walled girders with application to ship vibrations. Marine Structures, 2009, 22(3), p. 387-437.

9. Tomašević, S. Hydroelastic model of dynamic response of Container ships in waves. Doctoral Thesis, University of Zagreb, Zagreb, 2007, (in Croatian).
10. Pavazza, R. Torsion of thin-walled beams of open cross-sections with influence of shear. International Journal of Mechanical Sciences, 2005, 47, p. 1099-1122.

11. Senjanović, I., Tomašević, S, Rudan, S, Senjanović, T. Role of transverse bulkheads in hull stiffness of large container ships. Engineering Structures, 2008, 30, p. 2492-2509.

12. Senjanović, I., Vladimir, N., Tomić, M. The contribution of the engine room structure to the hull stiffness of large container ships. International Shipbuilding Progress, 2010, 57(1-2), 65-85.

13. Malenica, Š., Molin, B., Remy, F., Senjanović, I. Hydroelastic response of a barge to impulsive and non-impulsive wave load. Proc. Hydroelasticity in Marine Technology, Oxford, 2003, p. $287-$ 314.

14. Malenica, Š., Senjanović, I., Tomašević, S., Stumpf, E. Some aspects of hydroelastic issues in the design of ultra large container ships. Proc. IWWWFB, Plitvice Lakes, 2007.

15. Remy, F., Molin, B., Ledoux, A. Experimental and numerical study of the wave response of flexible barge. Proc. Hydroelasticity in Marine Technology, Wuxi, 2006, p. 255-264.

16. Senjanović, I. Grubišić, R. Coupled horizontal and torsional vibration of a ship hull with large hatch openings. Computers \& Structures, 1991, 41(2), p. 213-226.

17. Szilard, R. Theories and Applications of Plate analysis. John Wiley \& Sons, New York, 2004.

18. Bathe, KJ. 1996. Finite Element Procedures. Prentice Hall.

19. Senjanović, I. Finite Element Method in Ship Structures. University of Zagreb, Zagreb, 1998, (in Croatian).

20. Senjanović, I., Malenica, Š., Tomašević, S. Hydroelasticity of large container ships. Marine Structures, 2009, 22(2), p. 287-314.

21. Pedersen, PT. Torsional response of container ships. Journal of Ship Research, 1985, 31, p. 194-205.

22. Pedersen, PT. A beam model for the torsional-bending response of ship hulls. RINA Transactions, 1983, p. 171-182.

23. Haslum, K., Tonnessen, A. An analysis of torsion in ship hull. European Shipbuilding, 1972, (5/6), p. 67-89.

24. Malenica, Š. Some aspects of hydrostatic calculations in linear seakeeping. Proc. $14^{\text {th }}$ NAV Conf., Palermo, 2003.

25. Price, WG, Wu, Y. Hydroelasticity of Marine Structures. In: Theoretical and Applied Mechanics, FI. Niordson \& N. Olhoff, eds. Elsevier Science Publishers B.V., 1985, p. 311-337.

26. Newman, JN. Wave effects on deformable bodies. Applied Ocean Research, 1994, 16, p. 47-59.

27. Huang, LL., Riggs, HR. The hydrostatic stiffness of flexible floating structure for linear hydroelasticity. Marine Structures, 2000, 13, p. 91-106.

28. STIFF, User's manual, University of Zagreb, FAMENA, 1990. 